\title{
EL OSTION JAPONES (Crassostrea gigas) EN BAJA CALIFORNIA \\ Por:
}

\section{René İsias Oî̃vares}

Ciencias Marinas Vol. 2 Núm.l

\begin{abstract}
RESUMEN
En este escrito se mencionan algunos resultados obtenidos de un experimento que se llevó a cabo en la Unidad de Ciencias Marinas, bajo contrato con el Instituto Nacional de Pesca y la Secretaría de Recursos Hidráulicus.

Dicho experimento consistió an un cultivo piloto de ostión japonés (Crassostrea gigas). Este trabajo se llevó a cabo en la Bahía de San Quintin; los resultados obtenidos fueron altamente satisfactorios y representan una diversificación de la industria pesquera en Baja California.

Hasta la fecha de publicación de este artículo, la sociedad cooperativa Ensenada ha obtenido la concesión para explotar el ostión en la Bahía de San Quintín.
\end{abstract}

\section{ABSTRACT}

This paper refers to a project of the Instituto de Investigaciones Oceanologicas of the Universidad Autonoma de Baja California in Ensenada, Baja California, whose objectives are the different manners of diverting the traditional fishing activities in the State of Baja California. For that purpose, a Japanese oyster (Crassostrea gigas) pilot culture was established in the San Quintin Bay, Baja California.

In part of the project a description of the method used is included, as well as general data on the Japanese oyster growth.

\section{INTRODUCCION}

La industria pesquera en Baja California, ha canalizado sus actividades principalmente a la explotación de los recursos naturales tales como langosta, abulón, camarón, almeja y especies de escama. Según las estadísticas de producción estos recursos han sufrido una marcada disminución debido posiblemente a una sobreexplotación o falta de administración de estos recursos. Este problema ha repercutido principalmente sobre el sector cooperativista cuya tecnología se reduce únicamente a la explotación de sus recursos tradicionales de los mismos.

A fines de 1972, el Instituto de Investigaciones Oceanológicas dependiente de la Universidad Autónoma de Baja California, presentó un proyecio ante la Secretaría de Recursos Hidráulicos y el Instituto Nacional de Pesca, que consistía en la introducción del ostión japonés (Crassostrea gigas), Thunberg (1795) a la Bahía de -San Quintín, Baja California.
Este proyecto se elaboró en base a la situación de la industria pesquera así como a las opiniones de los diferentes sectores cooperativistas tendientes a la diversificación de las pesquerías.

La Bahía de San Quintín está localizada en la costa noroccidental de la Baja California entre los $30^{\circ} 24^{\prime} \mathrm{N}$ y $30^{\circ} 30^{\prime} \mathrm{N}$; $115^{\circ} 57^{\circ} \mathrm{C}$ y $116^{\circ} 0 \mathrm{l}^{\prime} \mathrm{W}$; la distancia por carretera desde Ensenada, Baja California es de $200 \mathrm{~km}$ aproximadamente. El fondo de la Bahía de San Quintín estó formado principalmente por limos, barros y lodos, existen además lugares que acusan cierta profundidad (aproximadamente $2.5 \mathrm{~m}$ ) y además protegidos del oleaje y la corriente.

En 1973 el Instituto de Investigaciones Oceanológicas llevó a cabo un estusdio que comprendía la hidrología de la Bahía de San Quintín durante invierno y primavera que demostró entre otros factores que no existía aparentemente factor limitante para el cultivo del ostión japonés Crassostrea gigas (Chávez y Alvarez, 1974).

El proyecto tenía como finalidad los 
siguientes aspectos:

1) Entrenamiento de personal en ostricultivo.

2) Determinar las variaciones hidrográficas de la Bahía de San Quintín.

3) Seleccionar una especie de ostión apropiado para la bahía.

4) Seleccionar el lugar adecuado para el cultivo tridimensional.

\section{METODO Y MATERIALES}

Las fijaciones de ostión japanés C. gigas, se obtuvieron en un laboratorio del Estado de Washington, con el fin de obtener semilla de alta calidad y libre de organismos que pudieran ser nocivos en nuestras aguas. Para la obtención de esta semilla, personal de la Escuela Superior de Ciencias Marinas, tuvo la oportunidad de utilizar las instalaciones de dicho laboratorio y mediante la aplicación de la técnica Milford obtuvieron 4,000 conchas madre, con un promedio de 47 fijaciones cuyas dimensiones variaban de 1 a 3 milímetros.

La semilla se transportó por avión hasta San Diego, Cal., de donde posteriormente se llevó a la Bahía de San Quintín, B. C., por carretera y se sembró en octubre de 1973.

El método de cultivo utilizado fue el de suspensión; consistió en 4 balsas con dimensiones de $3.60 \times 1.80 \mathrm{~m}$. Estas dimensiones se determinaron en base a la cantidad de semilla que se había obtenido. Para la construcción de las balsas se utilizó madera de segunda mano y como sistema de flotación 2 barras de styrofoam de $.30 \times .54 \times 2.70 \mathrm{~m}$.

Para la construcción de las sartas se utilizó alambre galvanizado Núm. 12, usando como espaciador una torcedura que mantenía separada la concha madre aproximadamente $20 \mathrm{~cm}$.

\section{RESULTADOS Y CONCLUSIONES}

Durante el desarrollo de este programa se tomaron datos de temperatura, salinidad, $\mathrm{pH}$ y algunos parámetros meteorológicos, los cuales demostraron que no había variaciones considerables que repercutieran en el crecimiento del ostićn. Aparentemente el factor dominante para los ostiones fue la temperatura, ya que se tomaron datos de crecimiento $y$ se relacionaron con las variaciones de temperatura, demostrando que existe un rango que permite un crecimiento continuo durante el invierno en la Bahía de San Quintín.

Se tomaron datos de crecimiento que mostraron claramente que no existe relación entre el tamaño de la concha y el crecimiento del organismo (Quayle, 1969).

Es importante mencionar el hecho de que el ostión cultivado por medio de balsas presentó una sobrevivencia de $30 \%$ $y$ además una continuidad en el crecimiento, lo cual significa una aparente ventaja sobre los lugares tradicionales de cultivo en el Estado de Washington, en E.U.A., ya que en estas zonas de cultivo el ostión se ve afectado por un período de hibernación.

Por lo que respecta a las posibilidades de introducir ostión japonés C. gigas a una escala comercial, es necesario que se lleven a cabo estudios que demuestren la productividad de la zona, ya que además presenta posibilidades de explota. ción turística, que en un momento dado será un factor limitante para el desarrollo de estos cultivos.

Entre las conclusiones que se obtuvieron de esta investigación, vale la pena mencionar el hecho de que es necesario se lleven a cabo más investigaciones de este ttpo, ya que el costo de la inversión es relativamente bajo y los resultados que se obtengan podrian llegar a solucionar grandes problemas.

\section{AGRADECIMIENTOS}

El autor desea expresar su agradecimiento a la Secretaría de Recursos Hidráulicos, al Instituto Nacional de Pesca por el soporte económico a esta investigación.

Además se agradece la valiosa cooperación al Dr. Víctor L. Loosanoff por la planeación del entrenamiento sobre ostricultivo y a la Fundación Janss por el soporte económico del mismo.

El presente trabajo fue presentado en el $V$ Congreso Nacional de Oceanografía efectuado en Guaymas, Son., en octubre de 1974.

\section{BIBLIOGRAFIA}

Chávez de Nishikawa Amelia, Saúl Alvarez Borrego. 1974. Hidrología de la Bahla de Son Quintín Baja California en Invierno y Primavera. Ciencias Marinas 1\{2\}. $31-62$.

Quayle, B. C. 1969. Pacific Oyster Culture in British Columbia Fisheries Research Boord of Canada, Ottawa. 